\title{
TREATMENT OF BENIGN PROSTATIC HYPERPLASIA
}

\author{
Jonathan W. Valvano, David Y. Yuan \\ Biomedical Engineering Program \\ University of Texas \\ Austin, Texas
}

\author{
Eric N. Rudie, Steven J. Clark \\ Urologix Inc. \\ Minneapolis, Minnesota
}

\begin{abstract}
The treatment of benign prostatic hyperplasia (BPH) has implications which affect the majority of the adult male population. Although benign compared to prostate cancer, clinical symptoms can dramatically alter the quality of life. The hyperplastic tissue can cause constriction of the urethra and thus affect voiding of urine. Factors to consider for thermally-based treatments of the prostate include minimization of thermal injury to the urethra and rectum, and maximal delivery of thermal energy to target tissue. Minimizing temperature rise in the urethra allows for minimal or no anesthesia, and has been shown to reduce postoperative complications. Protection of the rectal wall is imperative since injury can lead to clinical complications as severe as a rectal fistula. Due to its location immediately dorsal to the prostate, the ventral aspect of the rectal wall is susceptible to overheating when a uniform radiating microwave heat source is applied transurethrally to treat the prostate.

This paper summarizes the engineering technology, numerical modeling, and clinical results to date.
\end{abstract}

\section{INTRODUCTION}

Pathologic BPH is observed in approximately $50 \%$ of elderly men in their sixth decade, and the incidence increases to almost $100 \%$ by the eighth decade [1]. About half of these men will develop macroscopic enlargement of the gland, and clinical signs and symptoms will be manifested in about half of this population causing them to seek medical treatment. To be an effective treatment a therapy must address the underlying disease. Current surgical therapies accomplish this, however the complication rates turn many patients away from seeking treatments.

\section{MICROWAVE INSTRUMENTATION}

The Urologix ${ }^{\circledR}$ Transurethral Thermal Therapy $\left(\mathrm{T} 3{ }^{\circledR}\right)$ system addresses the need for an effective treatment with minimal complications. Our objective is to thermally necrose the hyperplastic tissue using controlled microwave energy while protecting the urethra and rectum from thermal injury. Microwave energy is delivered via a transurethral catheter with cold water flowing through subsurface lumens. The cold water flow minimizes temperature rise in the periurethral tissue which is located immediately adjacent to the catheter. Our catheter delivers energy to the prostate such that heat generation is greater towards the anterio-lateral aspects of the prostate. The treatment catheter produces the targeted heating by constructing the microwave antenna with an offset alignment. Because the $915 \mathrm{MHz}$ helical antenna is positioned above the center of the catheter, microwave power is preferentially directed into the ventral aspect of the prostate, away from the rectum. Cool water is forced through the catheter to prevent damage to the urethra. One lumen contains a temperature probe, another is used for the balloon, and a third lumen is for urine drainage.

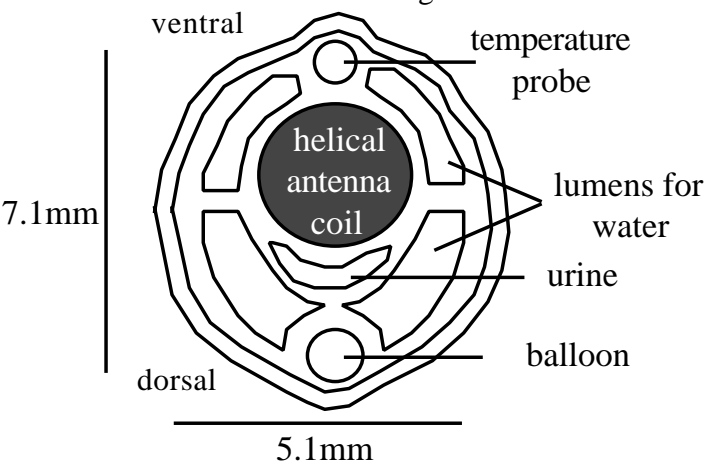

\section{CLINICAL STUDIES}

Interstitial temperature mapping of the prostate has been performed during microwave thermal treatments in 15 patients with $\mathrm{BPH}[3,4]$. The temperature measurement accuracy was better than $\pm 0.3^{\circ} \mathrm{C}$. The following figure shows a typical placement of Luxtron fiber optic temperature probes in the prostate. The probes were inserted using 17 gauge polymer afterloading needles containing metal obturators. The positions of the thermal sensors were verified using both ultrasonic and fluoroscopic imaging $( \pm 2 \mathrm{~mm}$.)

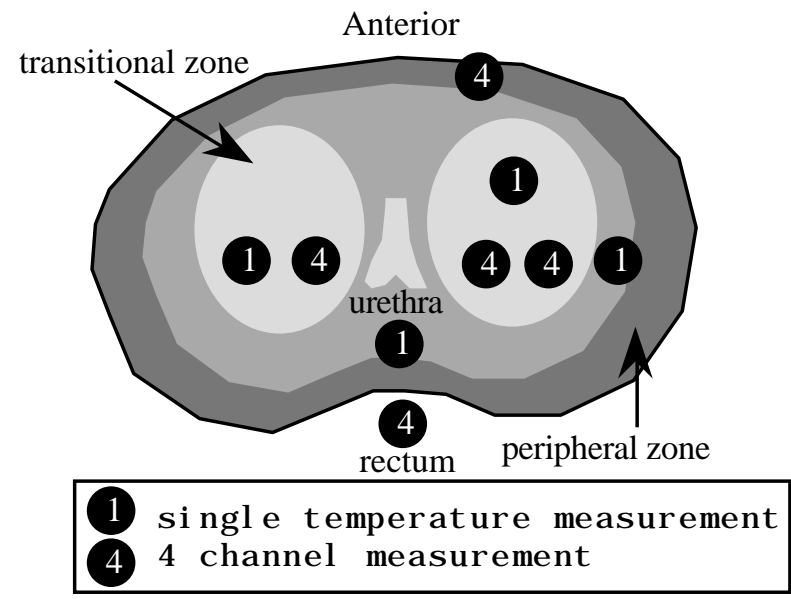


Microwave energy (average 32.9 watts) was delivered for about 1 hour to the prostate via the microwave antenna operating at $915 \mathrm{MHz}$ located in the Urologix catheter. The mean of the maximum temperature measured in the 15 patients is tabulated below:

\begin{tabular}{|lll|}
\hline location & \# of sites & maximum temperature \\
\hline prostate & 24 & $59.9 \pm 1.9^{\circ} \mathrm{C}$ \\
urethra & 1 & $39.6 \pm 0.9^{\circ} \mathrm{C}$ \\
rectum & 5 & $40.8 \pm 1.7^{\circ} \mathrm{C}$ \\
\hline
\end{tabular}

The study demonstrated the capability of the device to substantially elevate interstitial prostate temperature without raising urethral or rectal temperatures to uncomfortable or necrotic levels.

\section{NUMERICAL MODELLING}

The objective is to develop a realistic heat transfer model which predicts temperature elevations in the prostate generated by the T3 system. A two-dimensional finite element model, using Pennes' model for the heat transfer contribution of perfusion, was compared to the human interstitial temperature data. The finite element analysis enables the modeling of realistic tissue geometry's. Phantom experiments were used to develop a realistic microwave source term and boundary conditions for the catheter.

The cooling effect was modeled by treating the inner wall of the catheter as a convective boundary condition. The cooling effect was modeled by treating the inner wall of the catheter as a convective boundary condition:

$\mathrm{q}^{\prime \prime}$ cool $=\mathrm{h}\left(\mathrm{T}_{\text {coolant }}-\mathrm{T}_{\text {inner wall }}\right)$

where $\mathrm{q}^{\prime \prime}$ is the heat flux across the catheter inner wall surface, $\mathrm{h}$ is the convective cooling coefficient, and $\mathrm{T}$ is the temperature. This coefficient was derived empirically by comparing model with experimental results. Temperature data of a T3 catheter in phantom gel prior to heating was used. For the cooling experimental data, the model can agree to within about $\pm 2{ }^{\circ} \mathrm{C}$.

The microwave heat generation model represents the near field effect of an infinitely long microwave line source. This results in cylindrically shaped thermal energy deposition patterns centered at the antenna location. Microwave power absorption of the silicone catheter wall was assumed to be negligible compared to the heat transfer within the surrounding tissue. Therefore, only microwave power absorption in tissue was modeled. The heating term was modeled by:

$$
q^{\prime \prime \prime}(r, \theta)=A \frac{e^{-\beta r}}{r}
$$

As with deriving the cooling coefficient, results of a phantom gel experiment was used to derive the microwave coefficients. The antenna offset determines the anterior to posterior distribution of thermal energy whereas the microwave power coefficient (A) scales the amount of thermal energy deposition. The criteria for determining the best value for each coefficient $(A, h)$ is by comparing least square errors of data for all the probes at selected times. The effect of perfusion on heat transfer within the prostate is modeled by a temperature-dependent Pennes' :

$$
\rho c \frac{\partial T}{\partial t}=\nabla \cdot k \nabla T+(\rho c)_{b} \omega_{b}\left(T_{a}-T\right)+q_{m e t}
$$

where $\rho$ and $c$ are the tissue or blood density and specific heat, respectively, $\mathrm{t}$ is time, $\mathrm{k}$ is thermal conductivity, $\omega$ is perfusion, $\mathrm{T}_{\mathrm{a}}$ is the arterial blood temperature, and $\mathrm{qmet}_{\mathrm{me}}$ is metabolic heat generation (assumed to be negligible). A simple thermoregulatory response was assumed .

Three human interstitial temperature mapping data have been modeled. A model match with measured data to within $\pm 5{ }^{\circ} \mathrm{C}$ could be achieved by varying the perfusion temporally and spatially. The lack of measured blood flow response data necessitates empirically deriving a perfusion response model. A typical output of the model is shown in the following figure.

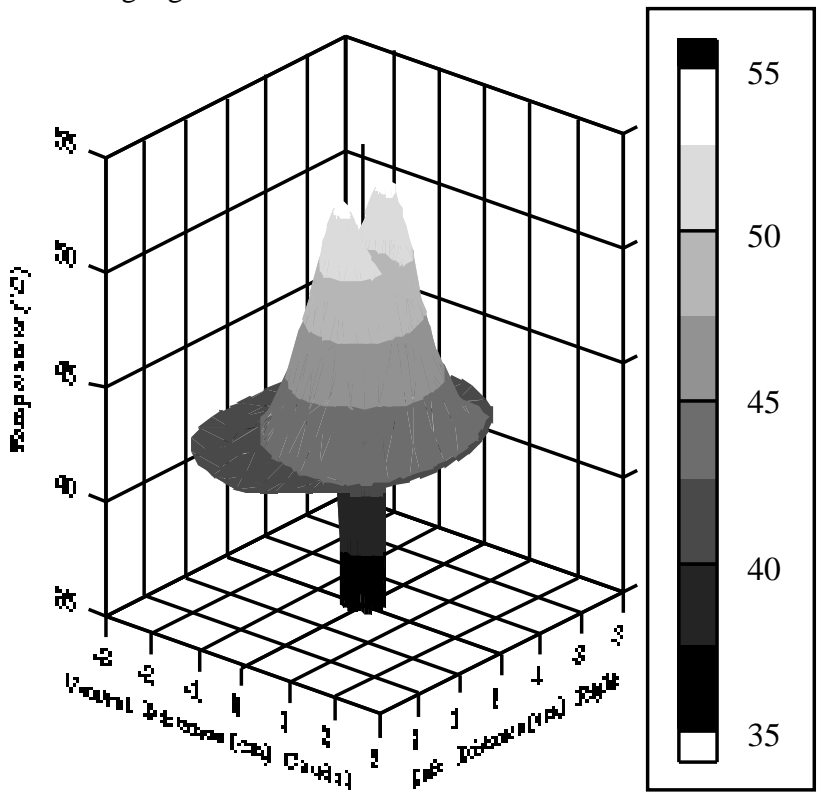

\section{CONCLUSIONS}

A transurethral catheter delivers microwave power to the prostate. The offset antenna and catheter cooling combine to create a device which applies heat to the anterio-lateral aspects of the prostate while protecting the urethra and rectum. The preferential heating design of the catheter was demonstrated by the non-uniform temperature distribution. Preliminary clinical studies and numerical models demonstrate the potential for this device to be an effective treatment of BPH.

\section{REFERENCES}

[1] J.T. Isaacs, and D.S. Coffey, "Etiology and Disease Process of Benign Prostatic Hyperplasia," The Prostate Supplement, 2: 33-50, 1989.

[2] L.X. Xu, E. Rudie, and K.R. Holmes, "Transurethral Thermal Therapy (T3) for the Treatment of Benign Prostatic Hyperplasia (BPH) in the Canine: Analysis Using Pennes Bioheat Equation," Proc. ASME Winter Annual Meeting, HTD-Vol. 268: 31 - 40, 1993.

[3] Larson, T.R., and Collins, J.M., "An Accurate Stereotactic technique for detailed prostatic interstitial temperature mapping in patients receiving microwave thermal treatment," J. Endourol., 9:339-347, 1995.

[4] Larson, T.R., and Collins, J.M., "Increased Prostatic blood flow in response to microwave thermal treatment: preliminary findings in two patients with benign prostatic hyperplasia," Urology, 46: 584-590, 1995. 